\title{
Network Forum Affects the Stock Market: An Empirical Analysis Based on Multivariate GARCH-BEKK Model
}

\author{
Hongbo Yía, Juanjuan Lai ${ }^{a}$, Dayong Donga, Guoen Xia ${ }^{\mathrm{b}}$
}

\begin{abstract}
Based on the text of posing on the network forum, the authors establish a set of keyword dictionary to measure the long and short investors' sentiment effectively, then they investigate the mutual relations between investor sentiment and the trading market through a Multivariable BEKK-GARCH model of abnormal long and short investor sentiment, returns, and abnormal trading volume. The results show that abnormal sentiment of long investor has a negative impact on the returns and a positive impact on the abnormal trading volume; while abnormal sentiment of short investor has no impact on the return and a negative impact on the abnormal trading volume. Otherwise, there is the negative volatility effect from abnormal sentiment of long investor to returns and abnormal trading volume, the positive volatility effects from abnormal sentiment of short investor to returns, and no volatility effects from abnormal sentiment of short investor to abnormal trading volume. In addition, network forum investor sentiment is a factor affecting the trading market. The analysis of forum information plays a certain role in presenting market risk and improving efficiency in making investment decision.
\end{abstract}

\section{Keywords}

Network forum, investor sentiment, sentiment volatility, trading market

Investor sentiment theory was first put forward by De Long. The investor sentiment, a significant topic in behavioral finance, aptly portrays investor's mental activities and reflects investor's expectation to market, which plays an important role in decision-making for investors. In the opinion of De Long et al. (1990), owing to the mutual influence and unpredictability of investor sentiments, irrationally pricing cannot be eliminated, so investor sentiments become systemic risk of financial assets' equilibrium price and affect returns and fluctuations. The research of Lee, Jiang, and Indro (2002) further proved that investor sentiments are systemic risk factors which influence prices. Zongxin Zhang and Hailiang Wang (2013) found that new information led to the adjustment of investors' subjective beliefs, which further influenced the change of investor sentiments and then reflected with the fluctuation of stock prices. Pessimism and optimism to stock markets are both included in investor sentiments. Since investor sentiments influence fluctuation of risks and returns in stock market, how do they play a role in affecting stock price and fluctuation? Which one leads market risk, pessimistic sentiment or optimistic sentiment?

\footnotetext{
aSouthwest Jiaotong University, Chengdu, China bGuangxi University of Finance and Economics, China

\section{Correspondent Author:}

Hongbo Yi, No. 111, North Erhuan Road, Chengdu, China E-mail: yihongboyunnan@126.com
} 
Sentiments are defined as a belief of wrong expectation to future cash flows and investment risk, which are caused by the irrelevant information about capital values in the capital market and by the future uncertainty. This kind of erroneous beliefs leads irrational behavior by noise traders and incorrectly pricing. Individual investors are always influenced by sentiments and then behavioral biases and cognitive biases occur during the process of trading. Analyzing investor sentiments has theoretical and practical significance in risk reduction and promoting a stable trading markets.

Zhigao Yi and Ning Mao (2009) thought that sentiments can promote investors to reach unanimity under the interactive mechanism and then influence investors' decision. According to the 34th China Internet Development Report by CNNIC (China Internet Network Information Center), by June 2006, the number of retail investors in China reached 632 million, the popularity rate of internet reached $46.9 \%$. As a social media providing platforms for retail investors to express opinions and get information, stock forums have strong interactivity, large quantities of information, fast update speed, so they can reflect the investors' responses to stock markets in time. Besides, retail investors can influence stock markets through posting and adding comments on the forum. Therefore, this paper will extract investors' sentiments based on forums and put them into the analysis of stock prices fluctuation and market risks, which is absolutely a significant breakthrough and innovation compared with the angle of traditional analysis.

In summary, this paper focuses on whether postings on forums measure different investor sentiments efficiently? Whether investor sentiments can influence the return and fluctuation in stock markets and how it works? Further more, whether two different investor sentiments, pessimistic sentiment or optimistic sentiment have the same effect on the fluctuation of stock price? Market risk comes from long investor or short investor? These problems are what this paper discusses.

\section{LITERATURE REVIEW}

Studies have shown that investor sentiments based on social media are well correlated with stock market. Tetlock (2007) linked comment column on Wall Street Journal with return and volume in stock market, and then found that the upper-middle-level pessimistic sentiments indicate price falling, but excessively high or low pessimistic sentiments indicate high volume. Fotak (2008) divided long and short suggestions on finance blogs in 2006 into long-term and short-term suggestions in order to study their effects on stock prices and trading volume, from which he found that suggestions on blogs could lead abnormal trading volume and negative abnormal return. Dayong Dong and Zuoping Xiao (2012) initially tentatively used information on forum to prove that there is bidirectional fluctuation spillover effect between returns in stock market and abnormal posting volume. However, Dong's main purpose is to test the information dissemination between trading market and stock forum, so he did not analyze contents of postings in detail. Therefore, there is no research on whether and how investor sentiments based on stock forums can affect market returns, trading volume, and fluctuations.

Foreign scholars have done some research on how to get sentimental information from large quantities of unstructured text information and their research results have provided theoretical and technical references and supports for this paper. Changqin Quan and Ren (2010) chose blogs as objects and data sources of expressing sentiments. Then, he constructed emotional corpus that have been annotated manually and studied expression of sentiments in Chinese. With experiments on expressing sentiments in texts, he found that emotional key words could reflect $69 \%$ of sentiments and the rest $31 \%$ of sentiments needed more analysis on grammar and semantics. O'Leary 
(2011) proposed to use readers' alternative labeling information to measure sentiments expressed in blogs rather than rely on labeling information provided by blogs themselves. Loughran and McDonald (2011) constructed an alternative negative words list and found that these words had significant correlation with financial variables such as daily return, trading volume, and fluctuation of return rate that day $10-\mathrm{k}$ companies declared, supporting that these words can effectively measure tones and sentiments.

Therefore, this paper makes an text analysis with postings on forums, establishes indexes that can effectively measure sentiment of long-short investors, and studies how do investor sentiments influence return and fluctuation. It is the first time to measure individual investor sentiments based on stock forum though text analysis techniques, and empirically analyze how do sentiments influence return and fluctuation in China stock market, which is the main innovation point of this paper.

\section{STUDY METHODOLOGY}

\section{Samples and Data}

This paper selects daily returns and daily trading volume of the Shanghai Composite Index as the basic data of market, which are from CSMAR (China Stock Market \& Accounting Research) database. The sample covers 884 trading days from January 7, 2010 to August 30, 2013. The sentiment of long-short investors is based on the text in the posing on Eastmoney forum bar about Shanghai Stock Exchange (SSE) Composite Index.

\section{The Establishment of Keyword Dictionary of "The Long Sentiment" and "The Short Sentiment" and Measuring Sentiment}

This paper downloads the content of posting on Eastmoney forum bar about SSE Composite Index from January 7, 2010 to August 30, 2013, by programming the web crawler. Then, the authors make a word segmentation and frequency statistics of the posting content to select the keywords that represent the investor sentiment and behavior artificially by the procedure of frequency statistics, 394 keywords in total. Finally, the authors put these words into two categories by repeatedly comparing. This paper defined these keywords that reflect negative sentiment such as pessimism, panic, anxious, and bearish as a dictionary of "the short sentiment", 202 keywords in total. On the contrary, the paper defined these keywords that reflect positive sentiment such as optimism, happy, promising, and bullish as a dictionary of "the long sentiment", 192 keywords in total.

\section{The Establishment of the Variables}

This paper discusses the lead-lag relationship that investor sentiment based on the forum have on the returns and the trading volume. Namely, the effect that the investor sentiments in one day have on the market in the next day. The daily trading volume, the sentiment of long-short investor are measured by the logarithmic volume and the logarithmic sentiment respectively as followed:

$$
\begin{gathered}
V o l_{t}=\ln \left(\text { vol }_{t}\right) \\
L_{-} \text {sent }_{t}=\ln \left(l_{-} \text {sent }_{t}\right) \\
S_{-} \text {sent }_{t}=\ln \left(s_{-} \text {snet }_{t}\right)
\end{gathered}
$$

The descriptive statistics are shown in Table 1. All the variables were tested at the $1 \%$ level of significance, indicating that all the series are stationary series, so they can apply to ARMA (autoregressive moving average) model. In the $\operatorname{ARMA}(p, q)$ model, the order of autoregressive (p) and moving average (q) is determined in line with AIC (Akaike information criterion) and SC (Schwarz criterion). The ARMA model and parameter estimation are as follows:

Model 1: The $\operatorname{ARMA}(2,1)$ model of trading volume $\left(\operatorname{Vol}_{t}\right)$ : 
Table 1. The Basic Descriptive Statistics on Variables

\begin{tabular}{llllllll}
\hline Variables & Mean & Std. dev. & Max. & Min. & Skewness & Kurtosis & ADF \\
\hline Vol $_{t}$ & 13.686 & .1146 & 14.783 & 12.899 & .1152 & -.2931 & $40.26[.001]$ \\
L_sent $_{t}$ & 4.9816 & .3698 & 6.4815 & 2.7080 & -.3711 & -.2555 & $69.52[.001]$ \\
S_sent $_{t}$ & 5.0102 & .5309 & 6.7440 & 2.3025 & -.2980 & -.1817 & $73.79[.001]$ \\
\hline
\end{tabular}

$$
\begin{aligned}
& \mathrm{Vol}_{t}=\mu_{V o l}+\phi_{11} \mathrm{Vol}_{t-1}+ \\
& \phi_{12} \operatorname{Vol}_{t-2}+\theta_{11} \varepsilon_{t-1}+\varepsilon_{V l_{t}}
\end{aligned}
$$

Model 2: The ARMA $(5,1)$ model of the long sentiment $\left(L_{-}\right.$sent $\left._{t}\right)$ :

$$
\begin{aligned}
& L_{-} \text {sent }_{t}=\mu_{L_{-} \text {sent }}+\phi_{21} L_{-} \text {sent }_{t-1}+ \\
& \phi_{22} L_{-} \text {sent }_{t-2}+\phi_{23} L_{-} \text {sent }_{t-3}+\phi_{24} L_{-} \text {sent }_{t-4}+ \\
& \phi_{25} L_{-} \text {sent }_{t-5}+\theta_{21} \varepsilon_{t-1}+\varepsilon_{L_{-} \text {sent }_{t}}
\end{aligned}
$$

Model 3: The $\operatorname{ARMA}(1,2)$ model of the short sentiment $\left(S_{-}\right.$sent $\left.t_{t}\right)$ :

$$
\begin{aligned}
& S_{-} \text {sent }_{t}=\mu_{S_{-} \text {sent }}+\phi_{31} S_{-} \text {sent }_{t-1} \\
& +\theta_{31} \varepsilon_{t-1}+\theta_{32} \varepsilon_{t-2}+\varepsilon_{S_{-} \text {sent } t_{t}}
\end{aligned}
$$

The regression results of Table 2 show that all the parameters pass the significant test. So the residual $\left(\varepsilon_{\text {Vol }_{t}}\right)$ is defined as abnormal trading volume $\left(A V o l_{t}\right)$, the residual $\left(\varepsilon_{L_{-} \text {sent }_{t}}\right)$ is defined as the abnormal sentiment of long investor $\left(A L_{-}\right.$sent $\left._{t}\right)$, and the residual $\left(\varepsilon_{S_{-} \text {sent }_{t}}\right)$ is defined as the abnormal sentiment of short investor $\left(S L \_\right.$sent $\left._{t}\right)$.

\section{Empirical Model}

This paper applied the multivariate GARCH-BEKK model to study the relationship among the four variables that are returns, abnormal trading volume and the abnormal sentiment of the long-short investors. BEKK model was proposed by Engle and Kroner (1995), whose advantage is to ensure that the covariance matrix should satisfy positive definite condition and less number of parameter estimation. This paper sets up quarternary BEKK model as follows:

$$
\begin{aligned}
& \left\{\begin{array}{l}
R_{t}=\mu_{r}+\gamma_{1} R_{t-1}+\eta_{1} A \text { Vol }_{t-1}+\kappa_{1} A L_{-} \text {sent }_{t-1} \\
+\lambda_{1} A S_{-} \text {sent }_{t-1}+\varepsilon_{1 t} \\
A V o l_{t}=\mu_{A V o l}+\gamma_{2} R_{t-1}+\eta_{2} A L_{-} \text {sent }_{t-1} \\
+\kappa_{2} A S_{-} \text {sent }_{t-1}+\varepsilon_{2 t} \\
A L_{-} \text {sent }_{t}=\mu_{A L_{-} \text {sent }}+\gamma_{3} R_{t-1}+\eta_{3} A \text { Vol }_{t-1} \\
+\kappa_{3} A S_{-} \text {sent }_{t-1}+\varepsilon_{3 t} \\
A S_{-} \operatorname{sent}_{t}=\mu_{A S_{-} \text {sent }}+\gamma_{4} R_{t-1}+\eta_{4} A \text { Vol }_{t-1} \\
+\kappa_{4} A L_{-} \operatorname{sent}_{t-1}+\varepsilon_{4 t}
\end{array}\right. \\
& \qquad H_{\mathrm{t}}=C^{\prime} C+A^{\prime} \varepsilon_{\mathrm{t}-1} \varepsilon_{\mathrm{t}-1}{ }^{\prime} A+B^{\prime} H_{t-1} B
\end{aligned}
$$

Where Equation (7) is the mean equation of BEKK model, Equation (8) is the variance equation. $R_{t}, A V o l_{t}, A L_{-}$sent $_{t}, A S_{-}$sent $_{t}$ and $\mu_{r}, \mu_{A V o l}, \mu_{A L_{-} \text {sent }}$, $\mu_{A S_{-} \text {sent }}$ represent daily logarithmic return, daily abnormal trading volume, daily abnormal sentiment of long investor, daily abnormal sentiment of long investor and the mean value of the four variables in the sample interval. $\varepsilon_{t}$ is $4 \times 1$ error vector, the corresponding conditional covariance matrix is $H_{t} . I_{t-1}$ represents information set at $t-1$ time. The residual hypothesis obeys multivariate GED (generalized error distribution) distribution. The variance Equation (8) is shown as above.

Where $\alpha_{i j}$ is the ARCH coefficient. When $i=j$, it represents the influence degree from the prior information to the market. When $i \neq j$, it represents the influence degree from the volatility of series $i$ to the volatility of series $j$, reflecting the ARCH effect of fluctuation. $\beta_{i j}$ is the GARCH (Generalized Autoregressive Conditional Heteroskedasticity) coefficient, representing the vitality of volatility 
transmission between series $i$ and series $j$, reflecting the GARCH effect of fluctuation.

$\left[\begin{array}{llll}h_{11, t} & h_{12, t} & h_{13, t} & h_{14, t} \\ h_{21, t} & h_{22, t} & h_{23, t} & h_{24, t} \\ h_{31, t} & h_{32, t} & h_{33, t} & h_{34, t} \\ h_{41, t} & h_{42, t} & h_{43, t} & h_{44, t}\end{array}\right]$
$=\left[\begin{array}{llll}c_{11} & 0 & 0 & 0 \\ c_{21} & c_{22} & 0 & 0 \\ c_{31} & c_{32} & c_{33} & 0 \\ c_{41} & c_{42} & c_{43} & c_{44}\end{array}\right]^{T}\left[\begin{array}{llll}c_{11} & 0 & 0 & 0 \\ c_{21} & c_{22} & 0 & 0 \\ c_{31} c_{32} & c_{33} & 0 \\ c_{41} & c_{42} & c_{43} & c_{44}\end{array}\right]$
$+\left[\begin{array}{llll}a_{11} & a_{12} & a_{13} & a_{14} \\ a_{21} & a_{22} & a_{23} & a_{24} \\ a_{31} & a_{32} & a_{33} & a_{34} \\ a_{41} & a_{42} & a_{43} & a_{44}\end{array}\right]^{T}\left(\begin{array}{llll}a_{11} & a_{12} & a_{13} & a_{14} \\ a_{21} & a_{22} & a_{23} & a_{24} \\ a_{31} & a_{32} & a_{33} & a_{34} \\ a_{41} & a_{42} & a_{43} & a_{44}\end{array}\right]$
$+\left[\begin{array}{llll}\beta_{11} & \beta_{12} & \beta_{13} & \beta_{14} \\ \beta_{21} & \beta_{22} & \beta_{23} & \beta_{24} \\ \beta_{31} & \beta_{32} & \beta_{33} & \beta_{34} \\ \beta_{41} & \beta_{42} & \beta_{43} & \beta_{44}\end{array}\right]^{T}\left[\begin{array}{llll}h_{11, t-1} & h_{12, t-1} & h_{13, t-1} & h_{14, t-1} \\ h_{21, t-1} & h_{22, t-1} & h_{23, t-1} & h_{24, t-1} \\ h_{31, t-1} & h_{32, t-1} & h_{33, t-1} & h_{34, t-1} \\ h_{41, t-1} & h_{42, t-1} & h_{43, t-1} & h_{44, t-1}\end{array}\right]\left[\begin{array}{llll}\beta_{11} & \beta_{12} & \beta_{13} & \beta_{14} \\ \beta_{21} & \beta_{22} & \beta_{23} & \beta_{24} \\ \beta_{31} & \beta_{32} & \beta_{33} & \beta_{34} \\ \beta_{41} & \beta_{42} & \beta_{43} & \beta_{44}\end{array}\right]$

\section{EMPIRICAL ANALYSIS}

\section{The Basic Descriptive Statistics on Variables}

The basic descriptive statistics, the auto correlation test, stationary test, and normality test are shown in Table 3. The normality test results (J-B) of $R_{t}, A V o l$, $A L \_$sent, and $A S_{-}$sent show that these four series refuse hypothesis of normal distribution. The test of Q-statistic shows that there is no auto correlation for these four variables. But the $\mathrm{Q}^{2}$-statistic shows that all the variables have auto correlation with a high order. That is to say, time-variant and aggregation exist in the fluctuation of all the variables. Besides, the ADF test shows that all the variables are stationary series.

\section{The Parameter Estimation of Quarternary BEKK-GARCH}

The parameter estimation is shown in Table 4. From the perspective of the mean equation of returns, abnormal trading volume is not significant at the $10 \%$ significance level, which illustrates that the abnormal trading volume cannot explain the returns. The regression coefficient of abnormal sentiment of long investor passes the significant test, which illustrates abnormal sentiment of long investor has an impact on the returns. The regression coefficient is negative, that is to say, the bullish expectation of investors based on network forum is not realized, the market will fall instead. It can be explained that in such a Chinese market which is lack of short system, there are games between individual investors and institutional investors, individual investors are often misleading by information asymmetry, cognitive bias, and herd behavior so that they are in the inferior position in the stock market. The regression coefficient of abnormal sentiment of short investor does not pass the significant test, which illustrates abnormal sentiment of short investor based on network forum has no impact on the returns, that is to say, the negative sentiment of investors on the forum can not affect the stock price.

From the perspective of the mean equation of abnormal trading volume, the parameter estimations of returns, abnormal sentiment of long investor, and abnormal sentiment of short investor pass the significant test at $1 \%$. The coefficient of return is positive, which indicates the returns have an positive impact on the abnormal trading volume. So the price rising in the market early can make investors to participate in the stock market actively, while a falling market make investors to have a cautious attitude. The positive abnormal sentiment of long investor indicates the abnormal sentiment of long investor early have a positive impact on the abnormal trading volume, that is to say, an optimistic sentiment expression will make investors to increase the demand for the stock. The negative abnormal sentiment of short investor indicates the abnormal sentiment of short investor early have a reverse impact on the abnormal trading volume.

From the perspective of the mean equation of abnormal sentiment of long investor, the parameter 
Table 2. The Regression Results of ARMA(p,q)

\begin{tabular}{lllllllll}
\hline & Constant & $\varphi_{i 1}$ & $\varphi_{i 2}$ & $\varphi_{i 3}$ & $\varphi_{i 4}$ & $\varphi_{i 5}$ & $\theta_{i 1}$ & $\theta_{i 2}$ \\
\hline Model 1 & 13.70926 & 1.31905 & -.3427 & & & & .70739 & \\
ARMA(2,1) & $(186.95)$ & $(16.89)$ & $(-4.75)$ & & & & & \\
& {$[.000]$} & {$[.000]$} & {$[.000]$} & & & & & \\
& & & & & & \\
Model 2 & 4.94809 & 1.32917 & -.15033 & -.22999 & .21253 & -.16369 & & \\
ARMA(5,1) & $(18.05)$ & $(29.35)$ & $(-2.66)$ & $(-4.17)$ & $(3.82)$ & $(-4.35)$ & $(32.42)$ & \\
& {$[.000]$} & {$[.000]$} & {$[.007]$} & {$[.000]$} & {$[.000]$} & {$[.000]$} & {$[.000]$} & \\
Model 3 & 5.00527 & .97174 & & & & & .48849 & .17140 \\
ARMA(1,2) & $(29.44)$ & $(100.73)$ & & & & & $(13.95)$ & $(4.94)$ \\
& {$[.000]$} & {$[.000]$} & & & & & {$[.000]$} & {$[.000]$} \\
\hline
\end{tabular}

Table 3. The Basic Descriptive Statistics on Variables

\begin{tabular}{lllll}
\hline & $R t$ & $A V o l$ & $A L_{-}$sent & $A S_{-}$sent \\
\hline Mean & -.0004 & .0009 & .0089 & .0051 \\
Std. dev. & .0001 & .0332 & .1128 & .1875 \\
Skewness & -.3186 & .6086 & -.1963 & -.0402 \\
Kurtosis & 1.8238 & 1.2548 & 8.2014 & 1.7592 \\
J-B & $137.32[.000]$ & $86.682[.000]$ & $106.65[.000]$ & $1,908.2[.000]$ \\
$Q(1)$ & $.1363[.712]$ & $.0003[.985]$ & $.0100[.920]$ & $.0015[.969]$ \\
$Q(5)$ & $4.5982[.467]$ & $.6158[.987]$ & $.2200[.999]$ & $1.7936[.877]$ \\
$Q(15)$ & $14.401[.495]$ & $5.9958[.980]$ & $12.474[.643]$ & $18.965[.215]$ \\
$\mathrm{Q}^{2}(1)$ & $.4122[.521]$ & $.0274[.868]$ & $12.211[.000]$ & $.3343[.563]$ \\
$\mathrm{Q}^{2}(5)$ & $7.4571[.189]$ & $4.1192[.532]$ & $34.876[.000]$ & $9.8889[.078]$ \\
$\mathrm{Q}^{2}(15)$ & $23.400[.076]$ & $28.740[.017]$ & $124.30[.000]$ & $76.476[.000]$ \\
$\mathrm{ADF}$ & $30.03[.000]$ & $444.27[.001]$ & $458.91[.001]$ & $445.21[.001]$ \\
\hline
\end{tabular}

Table 4. The Parameter Estimation of Quarternary BEKK-GARCH

\begin{tabular}{|c|c|c|c|c|}
\hline & Parameter estimation & Std. dev. & T-statistc & $\mathrm{P}$ \\
\hline$\mu_{r}$ & -.00204545 & .00038789 & -5.27325 & .000 \\
\hline$R_{t}\{1\}$ & .35418595 & .04578096 & 7.73653 & .000 \\
\hline $\mathrm{AVol}_{t}\{1\}$ & .00012225 & .00260049 & .04701 & .962 \\
\hline$A L_{-} \operatorname{sent}\{1\}$ & -.00234755 & .00139349 & -1.68465 & .092 \\
\hline$A S_{-} \operatorname{sent}\{1\}$ & .00084351 & .00117225 & .71956 & .471 \\
\hline$\mu_{A V o l}$ & .06232608 & .00627535 & 9.93189 & .000 \\
\hline$R_{t}\{1\}$ & 3.48190371 & .67612762 & 5.14977 & .000 \\
\hline$A L_{-}$sent $\{1\}$ & .08086496 & .03009224 & 2.68724 & .007 \\
\hline$A S \_\operatorname{sent}\{1\}$ & -.13608196 & .02330037 & -5.84033 & .000 \\
\hline$\mu_{A L_{-} \text {sent }}$ & .07906921 & .00923271 & 8.56403 & .000 \\
\hline$R_{t}\{1\}$ & -5.43313539 & 1.08334658 & -5.01514 & .000 \\
\hline $\mathrm{AVol}_{t}\{1\}$ & -.03084664 & .05789086 & -.53284 & .594 \\
\hline$A S \_\operatorname{sent}\{1\}$ & .07550491 & .02890841 & 2.61187 & .009 \\
\hline$\mu_{A S_{-} \text {sent }}$ & .08932383 & .01289389 & 6.92761 & .000 \\
\hline$R_{t}\{1\}$ & -12.83322507 & 1.14355392 & -11.22223 & .000 \\
\hline $\mathrm{AVol}_{t}\{1\}$ & .05303872 & .08811701 & .60191 & .547 \\
\hline$A L_{-}$sent $\{1\}$ & -00918758 & .03399685 & -.27025 & .786 \\
\hline
\end{tabular}


Table 4 continued

\begin{tabular}{|c|c|c|c|c|}
\hline & Parameter estimation & Std. dev. & T-statistc & $\mathrm{P}$ \\
\hline$c_{11}$ & .01178397 & .00069129 & 17.04644 & .000 \\
\hline$c_{21}$ & .02149176 & .01392456 & 1.54344 & .122 \\
\hline$c_{22}$ & .17804962 & .00730623 & 24.36957 & .000 \\
\hline$c_{31}$ & -.05235129 & .01436645 & -3.64400 & .000 \\
\hline$c_{32}$ & .15487170 & .01136423 & 13.62800 & .000 \\
\hline$c_{33}$ & .00002983 & .01407338 & .00212 & .998 \\
\hline$c_{41}$ & -.26518564 & .01906281 & -13.91115 & .000 \\
\hline$c_{42}$ & .07510377 & .01792891 & 4.18898 & .000 \\
\hline$c_{43}$ & .00001678 & .01633091 & .00103 & .999 \\
\hline$c_{44}$ & .00000563 & .01375785 & $4.09313 \mathrm{e}-004$ & .999 \\
\hline$\alpha_{11}$ & .69119804 & .07058109 & 9.79296 & .000 \\
\hline$\alpha_{12}$ & 3.66913087 & .84547511 & 4.33973 & .000 \\
\hline$\alpha_{13}$ & -4.66018553 & 1.22539897 & -3.80299 & .000 \\
\hline$\alpha_{14}$ & -15.84904623 & 1.79723443 & -8.81857 & .000 \\
\hline$\alpha_{21}$ & .02465036 & .00384415 & 6.41243 & .000 \\
\hline$\alpha_{22}$ & -.15949765 & .05112081 & -3.12001 & .001 \\
\hline$\alpha_{23}$ & -.61222330 & .06352979 & -9.63679 & .000 \\
\hline$\alpha_{24}$ & -.79797880 & .10124171 & -7.88192 & .000 \\
\hline$\alpha_{31}$ & .00211039 & .00231903 & .91003 & .362 \\
\hline$\alpha_{32}$ & .35555798 & .03362100 & 10.57547 & .000 \\
\hline$\alpha_{33}$ & .70718162 & .04383568 & 16.13256 & .000 \\
\hline$\alpha_{34}$ & .49326076 & .06513542 & 7.57285 & .000 \\
\hline$\alpha_{41}$ & -.00198764 & .00185142 & -1.07357 & .283 \\
\hline$\alpha_{42}$ & -.16821514 & .02784418 & -6.04130 & .000 \\
\hline$\alpha_{43}$ & -.39726140 & .03521656 & -11.28053 & .000 \\
\hline$\alpha_{44}$ & -.30231137 & .04884017 & -6.18981 & .000 \\
\hline$\beta_{11}$ & .40494406 & .11230546 & 3.60574 & .000 \\
\hline$\beta_{12}$ & .10342677 & 1.13737058 & .09093 & .927 \\
\hline$\beta_{13}$ & 6.88349429 & 1.23779963 & 5.56107 & .000 \\
\hline$\beta_{14}$ & 12.68898031 & 2.24185956 & 5.66002 & .000 \\
\hline$\beta_{21}$ & -.01042728 & .00953905 & -1.09312 & .274 \\
\hline$\beta_{22}$ & .32360023 & .11384797 & 2.84239 & .004 \\
\hline$\beta_{23}$ & -.40440739 & .10407616 & -3.88569 & .000 \\
\hline$\beta_{24}$ & .16109050 & .21272373 & .75728 & .448 \\
\hline$\beta_{31}$ & -.00574990 & .00163999 & -3.50606 & .000 \\
\hline$\beta_{32}$ & -.13810859 & .03022030 & -4.57006 & .000 \\
\hline$\beta_{33}$ & .76220086 & .02891354 & 26.36138 & .000 \\
\hline$\beta_{34}$ & -.18770798 & .03576986 & -5.24766 & .000 \\
\hline$\beta_{41}$ & .00853036 & .00132847 & 6.42119 & .000 \\
\hline$\beta_{42}$ & .03235920 & .02985749 & 1.08379 & .278 \\
\hline$\beta_{43}$ & .12301453 & .02885566 & 4.26310 & .000 \\
\hline$\beta_{44}$ & .91155474 & .03750820 & 24.30281 & .000 \\
\hline Shape & .28362396 & .00946325 & 29.97110 & .000 \\
\hline
\end{tabular}


estimations of return is significant at $1 \%$, but the abnormal trading volume is not significant, which indicate the ups and downs in the market will have an impact on the investors' sentiment, the abnormal trading volume information does not. All the regression coefficients are negative, indicating that the market rising will weaken sentiment's optimism, but also will reduce more pessimistic emotion expression. It can be explained that the market rise likely make investors to shift their focus, investors will participate in discussion such as fundamental or other information. From the perspective of the mean equation of abnormal sentiment of short investor, the parameter estimations of return is significant negative at $1 \%$, the market decline will make investor to express more pessimistic sentiment. It can be explained when the market is falling, investors can not find the real reason that market is down, then their discussions contain the abuse, sadness sentiment, and so on. The parameter estimation of abnormal sentiment of short investor is significant positive at $1 \%$, while the long investor sentiment does not pass significance test.

From the perspective of the variance equation, the coefficient of $\beta_{11}, \beta_{31}, \beta_{41}$ is significant at $1 \%$, and $\beta_{21}$ is not significant at $10 \%$, which indicate the return volatility is not only influenced by itself early fluctuations, but also influenced by abnormal investor sentiment fluctuation fluctuation. $\beta_{31}<0$, it means abnormal sentiment volatility of long investor will have an reverse impact on the return volatility, $\beta_{41}>0$, it means abnormal sentiment volatility of short investor will have an positive impact on the return volatility. That is to say, abnormal sentiment volatility of long investor can reduce the market fluctuations efficiently, and abnormal sentiment volatility of short investor can increase the market fluctuations efficiently. So abnormal sentiment of short investor based on forum is a factor that influence market risk. $\beta_{13}>0, \beta_{14}>0$, which mean the return volatility has a positive impact on the abnormal investor sentiment volatility, the change of return is an influence factor that make investors sentiment to change. $\beta_{13}>\beta_{14}$, which indicates the market volatility have more intense impact on the abnormal sentiment volatility of short investor than long investor.

\section{CONCLUSIONS}

This paper makes an text analysis with the posing on Eastmoney forum bar about SSE Composite Index, establishing a set of keyword dictionary to measure sentiment of long and short investor effectively, then the authors investigate the effect that the abnormal sentiment volatility of investors have on the market through a Multivariable BEKK-GARCH model of abnormal long and short investors sentiment, returns, and abnormal trading volume. The main conclusions are as follows:

(1) The abnormal sentiment of long investor based on forum has a negative impact on the returns and a positive impact on abnormal trading volume; the abnormal sentiment of short investor has a negative impact on abnormal trading volume and no impact on the returns. The returns have a negative influence on the abnormal sentiment of long and short investors, while the abnormal trading volume has no influence on the abnormal sentiment of long and short investors;

(2) The abnormal sentiment volatility of long investor based on forum has a negative impact on the volatility of returns and abnormal trading volume; the abnormal sentiment volatility of short investor based on forum has a positive impact on the volatility of returns and no impact on the volatility of abnormal trading volume. The volatility of returns has a positive impact on the abnormal sentiment volatility of long and short investor, while the volatility of abnormal trading volume has a negative impact on the abnormal sentiment volatility of long investor and no impact on the abnormal sentiment volatility of short investor. 
The empirical results show that the expression of investor sentiment on the forum can influence the stock market further. With the rapid development of the network, the influence will become deeper and deeper. As a developing country, the development of financial markets is not mature enough relative to the developed countries, and the system risk is relatively high, so this research is very important. From the point of risk supervision, the results of this paper can provide a new perspective of supervision to the regulators, provide supervision basis for regulating the information dissemination of network media. It is significant to the supervision of network media, financial market stability, and the healthy development of the capital market. About the practical significance of the research, it can provide decision-making basis and an effective research method to the institutional and individual investors.

\section{Funding}

This paper is financed by the key projects: National Natural Science Foundation of China (Project No. 71271174) and Guangxi Province Universities and Colleges Excellence Scholar and Innovation Team Funded Scheme.

\section{References}

Delong, J. B., A. Shleifer, L. Summers, and R. J. Waldmann. 1990. "Noise Trader Risk in Finance." Journal of Political Economy 98(4):703-738.

Dong, D. Y. and Z. P. Xiao. 2012. "Is There Information Transmission Between Market and Internet Forum?" Management Review 23(11):3-11.

Engle, R. F. and K. F. Kroner. 1995. "Multivariate
Simultaneous Generalized ARCH.” Econometric Theory 11(1):122-150.

Fotak, V. 2008. "The Impact of Blog Recommendations on Security Prices and Trading Volume." Social Science Research Network Working Paper Series 15.

Lee, W. Y., C. X. Jiang, and D. C. Indro. 2002. "Stock Market Volatility, Excess Returns, and the Role of Investor Sentiment." Journal of Banking \& Finance 26(6):2277-2299.

Loughran, T. and B. McDonald. 2011. "When Is a Liability not a Liability? Textual Analysis, Dictionaries, and 10-Ks." The Journal of Finance 66(1):35-65.

O'Leary, D. E. 2011. "Blog Mining-review and Extensions: From Each According to His Opinion." Decision Support Systems 51(4):821-830.

Quan, C. and F. Ren. 2010. "A Blog Emotion Corpus for Emotional Expression Analysis in Chinese." Computer Speech \& Language 24(4):726-749.

Tetlock, P. C. 2007. "Giving Content to Investor Sentiment: The Role of Media in the Stock Market." The Journal of Finance 62(3):1139-1168.

Yi, Z. G. and N. Mao. 2009. "Study on Emotional Measurement of China Investors: The Construction of CICSI.” Journal of Financial Research 11:174-184.

Zhang, Z. X. and H. L. Wang. 2013. "Investor Sentiment, the Subjective Belief Adjustment and Market Volatility." Journal of Financial Research 4:142-155.

\section{Bios}

Hongbo Yi, Ph.D., Department of Finance and Accounting, Southwest Jiaotong University; research field: financial market. Juanjuan Lai, M.B.A., Department of Finance and Accounting, Southwest Jiaotong University; research field: financial market.

Dayong Dong, Ph.D., professor, Department of Finance and Accounting, Southwest Jiaotong University; research field: financial market.

Guoen Xia, Ph.D., professor, Department of Academic Affairs, Guangxi University of Finance and Economics; research field: financial market. 